\title{
Pushing periodic-disorder-induced phase matching into the deep-ultraviolet spectral region: theory and demonstration
}

\author{
Mingchuan Shao ${ }^{1}$, Fei Liang ${ }^{1}$, Haohai $Y^{1}$ and Huaijin Zhang ${ }^{1}$
}

\begin{abstract}
Nonlinear frequency conversion is a ubiquitous technique that is used to obtain broad-range lasers and supercontinuum coherent sources. The phase-matching condition (momentum conservation relation) is the key criterion but a challenging bottleneck in highly efficient conversion. Birefringent phase matching (BPM) and quasiphase matching (QPM) are two feasible routes but are strongly limited in natural anisotropic crystals or ferroelectric crystals. Therefore, it is in urgent demand for a general technique that can compensate for the phase mismatching in universal nonlinear materials and in broad wavelength ranges. Here, an additional periodic phase (APP) from order/ disorder alignment is proposed to meet the phase-matching condition in arbitrary nonlinear crystals and demonstrated from the visible region to the deep-ultraviolet region (e.g., $\mathrm{LiNbO}_{3}$ and quartz). Remarkably, pioneering 177.3-nm coherent output is first obtained in commercial quartz crystal with an unprecedented conversion efficiency above $1 \%$. This study not only opens a new roadmap to resuscitate those long-neglected nonlinear optical crystals for wavelength extension, but also may revolutionize next-generation nonlinear photonics and their further applications.
\end{abstract}

\section{Dear Editor,}

In 1961, nonlinear second-harmonic generation (SHG) was first discovered in quartz crystal ${ }^{1}$. In nonlinear parametric process, the phase-matching condition (the momentum relation between the fundamental and harmonic light) is indeed the most critical, corresponding to a constructive interference enhancement in a nonlinear medium and high-efficiency generation proportional to the crystal length ${ }^{2}$. Currently, associated with the natural birefringence ${ }^{3}$ of nonlinear crystals at typical angles (Fig. 1a) and periodic/aperiodic poled ferroelectric domains ${ }^{4,5}(+\boldsymbol{P}$ and $-\boldsymbol{P}$ ) (Fig. 1b) in certain nonlinear crystals ${ }^{6-8}$, coherent generation ranging from the visible region to the terahertz region has been developed and utilized in classical and quantum regions ${ }^{9-11}$. However, most nonlinear optical crystals have neither sufficient birefringence nor

Correspondence: Haohai Yu (haohaiyu@sdu.edu.cn) or

Huaijin Zhang (huaijinzhang@sdu.edu.cn)

${ }^{1}$ State Key Laboratory of Crystal Materials and Institute of Crystal Materials,

Shandong University, Jinan 250100, China controllable ferroelectric domains. Therefore, it is in urgent demand for the development of a new route to achieve phase matching in arbitrary nonlinear crystals and in broad wavelength ranges.

Deep-ultraviolet (DUV) coherent light is a fundamental source for an angle-resolved photoemission system ${ }^{12}$, photolithographic techniques ${ }^{13}$, and Raman spectroscopy ${ }^{14}$. The generation of DUV light by nonlinear frequency conversion is considered to be the "Holy Grail" in nonlinear optics $^{15}$. To the best of our knowledge, only potassium beryllium fluoroborate $\mathrm{KBe}_{2} \mathrm{BO}_{3} \mathrm{~F}_{2}(\mathrm{KBBF})$ nonlinear crystal has achieved direct birefringent phase-matchable SHG in the DUV region ${ }^{16}$. However, the wide application of KBBF crystals is limited by the great difficulty of crystal growth and toxicity of the raw material $\mathrm{BeO}^{17}$. In addition, random quasi-phase matching has also been realized in $\mathrm{SrB}_{4} \mathrm{O}_{7}$ crystal using a spontaneous domain structure ${ }^{18}$. However, the SHG conversion efficiency is too low (only $0.04 \%$ ) to be widely applied.

\section{(c) The Author(s) 2020}

(c) (i) Open Access This article is licensed under a Creative Commons Attribution 4.0 International License, which permits use, sharing, adaptation, distribution and reproduction cc) in any medium or format, as long as you give appropriate credit to the original author(s) and the source, provide a link to the Creative Commons license, and indicate if changes were made. The images or other third party material in this article are included in the article's Creative Commons license, unless indicated otherwise in a credit line to the material. If material is not included in the article's Creative Commons license and your intended use is not permitted by statutory regulation or exceeds the permitted use, you will need to obtain permission directly from the copyright holder. To view a copy of this license, visit http://creativecommons.org/licenses/by/4.0/. 
a
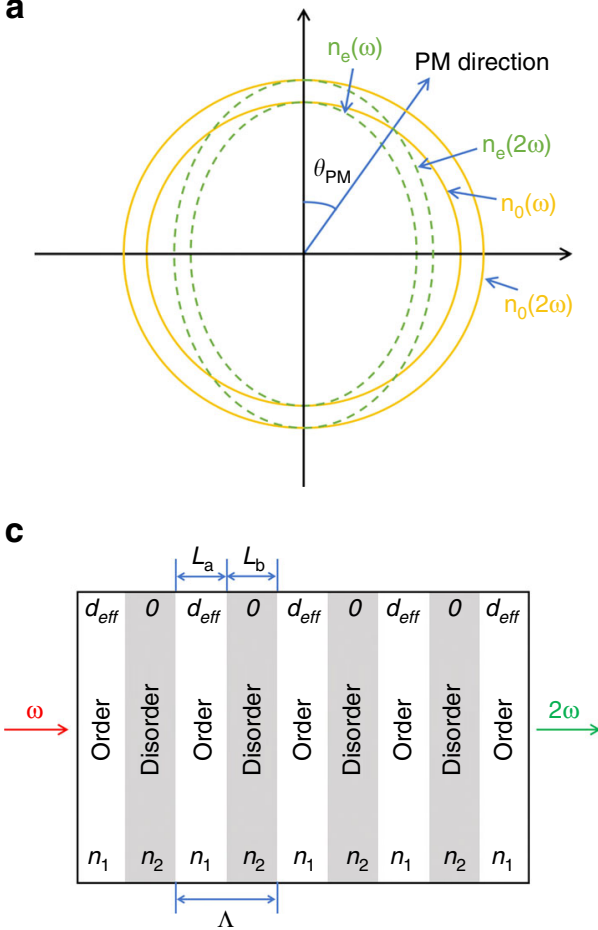

b

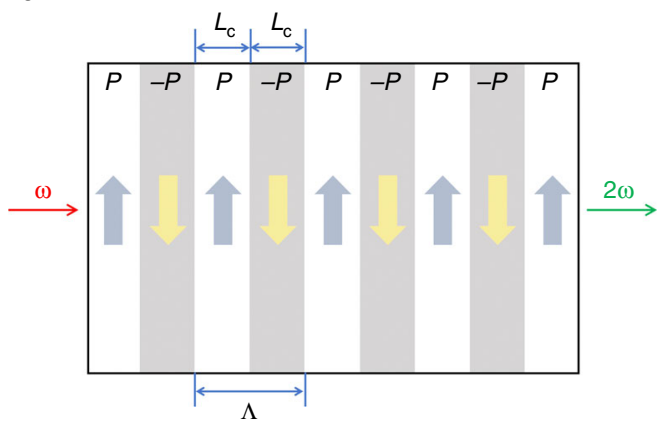

d

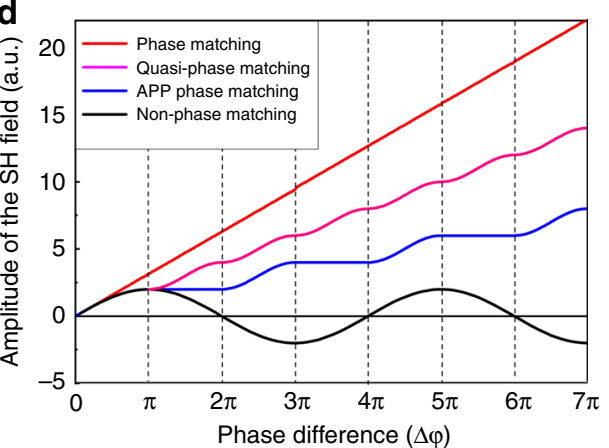

Fig. 1 APP phase matching for nonlinear frequency conversion. a Schematic graph of the birefringent phase-matching condition in a negative uniaxial crystal. $\theta_{P M}$ is the phase-matching angle. Along this direction, $n_{0}(\omega)=n_{e}(2 \omega)$ and $\Delta k=0$. $\mathbf{b}$ Schematic graph of the quasi-phase-matching condition in a ferroelectric crystal. The up and down arrows represent positive and negative $\boldsymbol{P}$ polarizations, respectively. The period length $\Lambda$ is double the coherence length $L_{c}\left(\Lambda=L_{c}+L_{c}\right)$. c Schematic graph of the additional phase-matching condition in arbitrary nonlinear optical crystals. The white and gray regions represent the ordered crystal and the disordered amorphous state, respectively. The period length $\wedge$ equals the sum of the ordered width $L_{a}$ and disordered width $L_{b}\left(\Lambda=L_{a}+L_{b}\right)$. Notably, $L_{a}$ and $L_{b}$ may be equivalent to $L_{c}$ or an integer multiple of $L_{c}$. $d_{\text {eff }} / 0$ and $n_{1} / n_{2}$ represent the second-order nonlinear coefficient and refractive index of the ordered and disordered regions, respectively. $\mathbf{d}$ The amplitude of the generated second-harmonic (SH) field under phase-mismatching and different phase-matching conditions. Equal interaction lengths of the nonlinear media and the same efficient nonlinear coefficients $d_{\text {eff }}$ are assumed. The period length $\wedge$ for quasi-phase-matching and additional phase matching is $2 L_{c}\left(\wedge=L_{c}+L_{c}\right)$

Herein, we propose an original concept, the additional periodic phase (APP) from order/disorder alignment (Fig. 1c), which can compensate for the mismatched phase in arbitrary nonlinear optical crystals. Moreover, the efficient SHG output in the visible, ultraviolet and even deepultraviolet regions is experimentally demonstrated in $\mathrm{LiNbO}_{3}$ and quartz. Remarkably, 177.3-nm coherent output is obtained in a quartz crystal with an unprecedented conversion efficiency above $1 \%$.

Taking the typical collinear frequency-doubling as an example, the electric field $E_{2 \omega}(z)$ of SHG light is described $\mathrm{as}^{2}$ :

$$
\frac{d E_{2 \omega}(z)}{d z}=\frac{2 i \omega}{c n_{2 \omega}(z)} d_{\mathrm{eff}}(z) E_{\omega}^{2} e^{-i \Delta \phi}
$$

where $E_{\omega}(z)$ denotes the electric field of the fundamental field at the propagation length $z$; $\omega$ refers to the fundamental frequency; $c$ represents the light velocity; $n_{2 \omega}(z)$ and $d_{\text {eff }}(z)$ denote the refractive indices of the SHG light and effective nonlinear coefficient at the propagation length $z$, respectively; and $\Delta \phi=\Delta k z=\left(k_{2}-2 k_{1}\right) z$ is the phase difference between the fundamental and SHG lights with the wavevectors $k_{1}$ and $k_{2}$, respectively.

Under the phase-mismatching condition, the amplitude of the SHG electric field will oscillate with a phase difference of $2 \pi$ in one period (black line in Fig. 1d). There is no effective coherent output under the phasemismatching condition except for a weak SHG signal. Assuming that the phase-matching condition is perfectly satisfied, the $\mathrm{SH}$ field amplitude consequently grows linearly with the phase difference (red line in Fig. 1d). In a ferroelectric crystal, quasi-phase matching can be achieved by adding the reciprocal vector $(G)$ with periodic reversal polarization (the sign of $d_{\text {eff }}$ ) to satisfy $\Delta k_{z}=k_{z}^{2}-2 k_{z}^{1}-m G=0$, where $m$ is an integer. Consequently, the generated $\mathrm{SH}$ field can continuously increase along the propagation direction (pink line in 
Fig. 1d). Compared with birefringent phase matching, quasi-phase matching is not limited by the special direction so as to use the largest $d_{\text {eff }}$ in certain nonlinear crystals, e.g., PPLN and PPKTP.

In accordance with the theory of quasi-phase matching, an additional periodic phase (APP) $\Delta \phi_{\mathrm{APP}}$, resulting from the alignment of order/disorder (crystal/amorphous) species, is proposed to compensate for the phase difference $\Delta \phi_{\mathrm{PD}}$ between the fundamental and SHG lights. The APP concept means that after the light propagates over the coherence length $L_{\mathrm{c}}$, the generated phase difference $\Delta \phi_{\mathrm{PD}}$ is compensated by the additional phase difference $\Delta \phi_{\text {APP }}$ with $\Delta \phi_{\mathrm{APP}}+\Delta \phi_{\mathrm{PD}}=0$ or $2 m \pi$ ( $m$ is an integer). The APP can be realized by periodically engineering regions in nonlinear crystals to undermine the translational symmetry of the nonlinear crystal and block the conversion of the energy from the SHG to the fundamental light, in addition to the oscillation of the SHG electric amplitude (blue line in Fig. 1d). The detailed mathematical analysis about the APP concept is presented in the Supplementary Information. In non-phase-matching condition, the APP of $\Delta \phi_{\mathrm{APP}}=(2 m+$ 1) $\pi$ and $\Delta \phi_{\mathrm{APP}}=2 m \pi$ will introduce constructive and destructive contributions to the SHG signal, respectively. Nevertheless, in our proposed APP strategy, the positive contribution from $\Delta \phi_{\mathrm{APP}}=(2 m+1) \pi$ remains, but the contribution from $\Delta \phi_{A P P}=2 m \pi$ decreases to zero, not a negative value. We can attribute the APP to the periodic variation of refractive index $\left(n_{1}\right.$ in the crystalline region and $n_{2}$ in the amorphous region) and effective nonlinear coefficient $d_{i j}\left(d_{\text {eff }}\right.$ in the crystalline region and zero in amorphous region). The periodic length $\Lambda$ is the sum of the coherent length $L_{\mathrm{a}}$ and the coherent length $L_{\mathrm{b}}$. Accordingly, an additional reciprocal vector $G=\frac{2 \pi}{\Lambda}$ is introduced. When $G=\Delta k$, the APP phase matching can achieve efficient SHG output, revealing that we can assume the proposed APP phase matching to be a new type of quasi-phase matching. In contrast to traditional quasi-phase matching based on the reversal domains in a few ferroelectric crystals, the proposed APP phase matching exhibits remarkable advantages in relaxing the phase-matching requirements and utilizing the largest efficient nonlinear coefficient $d_{\text {eff. }}$. Accordingly, this new APP phase-matching concept should be applicable to all non-centrosymmetric crystals to achieve highly efficient SHG output, even crystals without sufficient birefringence or reversible ferroelectric domains.

To experimentally demonstrate APP phase matching in nonlinear optical crystals, techniques to fabricate photonic crystals (e.g., laser writing processing and ion beam etching)

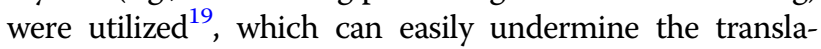
tional symmetry of a nonlinear crystal and generate amorphous regions in the nonlinear crystal, as shown in Fig. 1c. In this scenario, if the propagation length $L_{\mathrm{b}}$ of the light in the amorphous regions is controlled, the phase difference $\Delta \phi_{\mathrm{PD}}$ generated in the crystalline region can be compensated according to the introduction of the APP with $\Delta \phi_{\mathrm{APP}}+\Delta \phi_{\mathrm{PD}}=0$ or $2 m \pi$, only when $\Delta \phi_{\mathrm{PD}} \neq 2 \pi$.

First, a 1064-nm SHG experiment was performed in both ferroelectric $\mathrm{LiNbO}_{3}$ and non-ferroelectric quartz. A Nd:YAG laser (100 ns, $20 \mathrm{kHz}$ ) illuminated an as-prepared sample by a focusing lens $(f=100 \mathrm{~mm})$, and the SHG power was collected by a power meter (Fig. 2a). In previous studies, $\mathrm{LiNbO}_{3}$ and quartz are two common media used for optical waveguides in integrated photonics. With a suitable writing energy, amorphous $\mathrm{LiNbO}_{3}$ and quartz regions could be produced with a tunable period. A 350-fs pulsed laser at $1040 \mathrm{~nm}$ was used for the lasing writing. The $\mathrm{LiNbO}_{3}$ and quartz crystals were cut along the $\mathrm{X} / \mathrm{Z}$ direction to use the largest SHG coefficient $d_{33} / d_{11}$ in the nonlinear optical process. The periodic lengths $\Lambda$ of $\mathrm{LiNbO}_{3}$ and quartz are $6.79 \mu \mathrm{m}$ and $41.6 \mu \mathrm{m}$ for 1064-nm SHG conversion, respectively. By studying the grating diffraction of the samples based on Bragg diffraction (Supplementary Fig. S1), the refractive index divergence $\left(\Delta n=n_{1}-n_{2}\right)$ between crystalline and amorphous regions is very small, only $0.003-0.004$ in $\mathrm{LiNbO}_{3}$ and 0.004-0.005 in quartz from 350 to $630 \mathrm{~nm}$ (Fig. 2b). This is consistent with the reported conclusions in laserwritten $\mathrm{LiNbO}_{3}{ }^{20}$ and $\mathrm{SiO}_{2}$ waveguides ${ }^{19}$, suggesting that laser writing would not greatly break the dispersion of the refractive index. In addition, the broken translational symmetry in the written regions was also studied using the conoscopic interference technique (Supplementary Fig. S3). The results reveal that no obvious birefringence emerged along the $\mathrm{X}$ direction in the amorphous regions, indicating isotropic propagation of the fundamental and SHG waves. We have also demonstrated the SHG signal of the APP $\mathrm{SiO}_{2}$ sample with a second-harmonic microscope. The beam with a wavelength of $1030 \mathrm{~nm}$ is focused inside the quartz crystal through an objective lens (Fig. 2c). The upper part of the crystal is unprocessed, while the lower part is a processed periodic grating. There is no SHG signal for phase mismatching in the unprocessed region. In contrast, we can see a clear SHG signal in the region of the periodic grating, which proves the feasibility of the APP theory.

Figure $2 \mathrm{~d}$ shows the transmission spectra of the asgrown crystals and APP $\mathrm{LiNbO}_{3}$ samples processed with different writing energy intensities. The result suggests that the transparent cutoff edges of the prepared $\mathrm{LiNbO}_{3}$ samples are almost unchanged at $325 \mathrm{~nm}$. However, their transmission gradually reduces with increased writing energy intensity and becomes lower than 50\% under an energy intensity of $400 \mathrm{~J} / \mathrm{cm}^{2}$. Using APP $\mathrm{LiNbO}_{3}$ with $\Lambda=6.79 \mu \mathrm{m}$ and $\Delta \phi_{\mathrm{APP}}=\Delta \phi_{\mathrm{PD}}=\pi$ at the fundamental wavelength of $1064 \mathrm{~nm}$, the frequency-doubling performance at $532 \mathrm{~nm}$ (insert graph in Fig. 2e) was studied. The significant SHG output power $P_{2 \omega}$ is $26.11 \mathrm{~mW}$ at $532 \mathrm{~nm}$ for a pump power $P_{0}$ of $1260 \mathrm{~mW}$ (Fig. 2e). The 


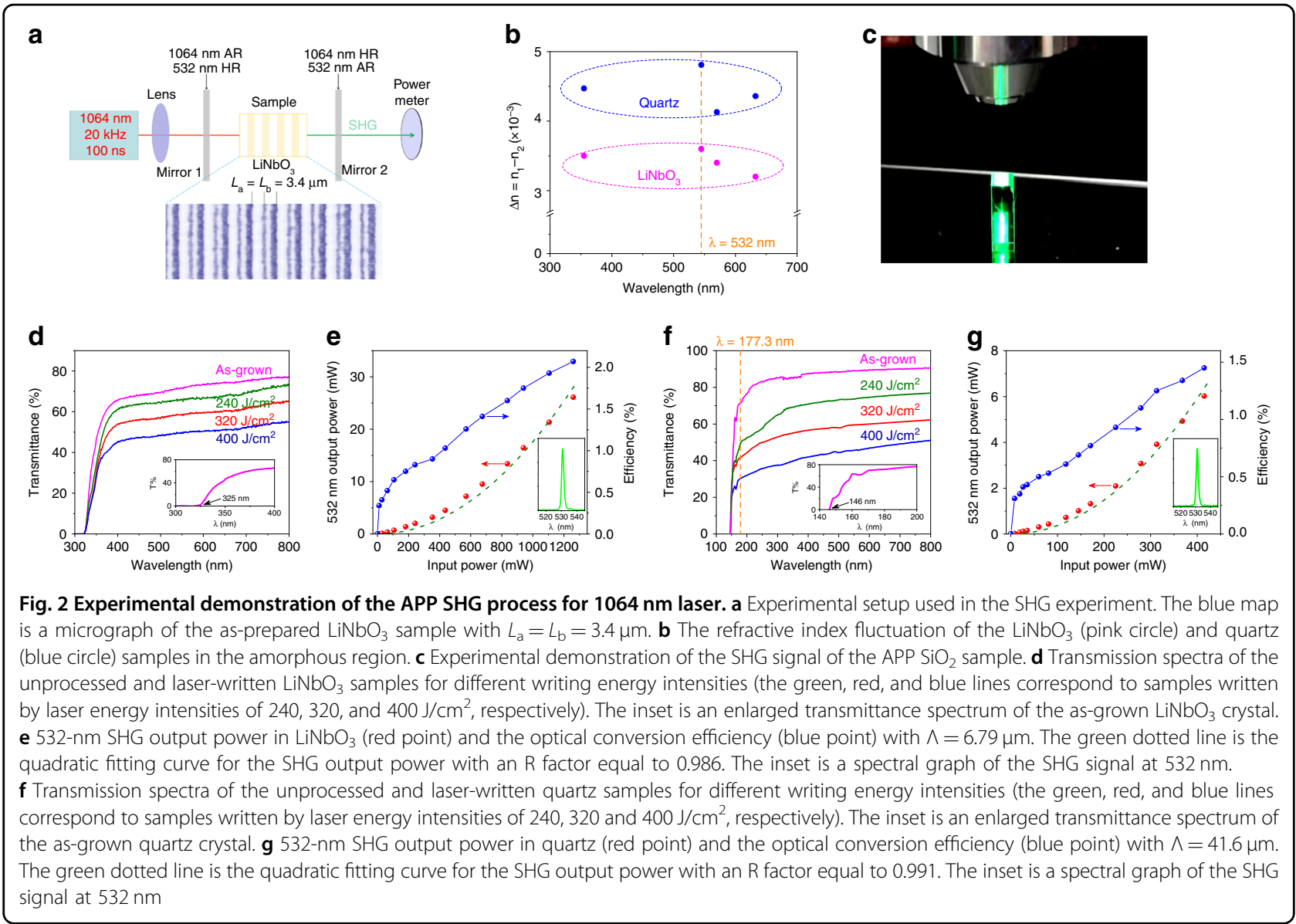

optical conversion efficiency $\eta\left(\eta=P_{2 \omega} / P_{0}\right)$ is $2.07 \%$. Meanwhile, quartz samples exhibit a shorter transmittance edge of $146 \mathrm{~nm}$ and a gradually reduced transmission with an increased writing energy intensity (Fig. 2f). The frequency-doubling signal at $532 \mathrm{~nm}$ is also detected in APP quartz (insert graph in Fig. 2g). The preliminary SHG output power $P_{2 \omega}$ is $6.02 \mathrm{~mW}$ at $532 \mathrm{~nm}$ for a pump power $P_{0}$ of $415 \mathrm{~mW}$ (Fig. $2 \mathrm{~g}$ ). Notably, as a nonbirefringent-matched nonlinear optical crystal, the conversion efficiency $\eta$ in APP quartz is calculated to be $1.45 \%$, which is approximately two orders of magnitude higher than the recently reported SHG result in a welldesigned silica microcavity $(\sim 0.049 \%)^{21}$ and in periodic stacking quartz plates $(\sim 0.01 \%)$ via traditional quasi-phase matching ${ }^{22}$. This result strongly demonstrates that our proposed APP phase-matching strategy is indeed effective.

Next, we demonstrated the APP strategy in the ultraviolet and solar-blind regions. Limited by the redshifted cutoff edge of $\mathrm{LiNbO}_{3}$, we only used quartz as an example. Two different periodically broken regions were designed in APP quartz with lengths of $L_{\mathrm{a}}=L_{\mathrm{b}}=2.1 \mu \mathrm{m}$ (sample A) and $1.4 \mu \mathrm{m}$ (sample B), corresponding to an APP of $\Delta \phi_{\mathrm{APP}}$ and a phase difference of $\Delta \phi_{\mathrm{PD}} \Delta \phi_{\mathrm{APP}}=\Delta \phi_{\mathrm{PD}}=\pi$ at SHG wavelengths of $242 \mathrm{~nm}$ and $214 \mathrm{~nm}$, respectively. Meanwhile, samples $\mathrm{A}$ and $\mathrm{B}$ are also relative to $\Delta \phi_{\mathrm{APP}}=$ $\Delta \phi_{\mathrm{PD}}=3 \pi$ and $2 \pi$ at the SHG wavelength of $177.3 \mathrm{~nm}$ (Fig. 3a), respectively. As mentioned above, the optical conversion efficiency strongly depends on the fluctuation of the phase difference. Only $\Delta \phi_{\mathrm{APP}}=\Delta \phi_{\mathrm{PD}}=(2 m+1) \pi$ is required to satisfy the phase-matching criteria, but $\Delta \phi_{\mathrm{APP}}=\Delta \phi_{\mathrm{PD}}=2 m \pi$ still maintains the non-phasematching condition. Remarkably, $\Delta \phi_{\mathrm{APP}}=\Delta \phi_{\mathrm{PD}}=\pi$ exhibits the highest SHG conversion efficiency for the same crystal length (Fig. 3b). An optical parametric oscillation laser with a pulse width of $10 \mathrm{~ns}$ was employed in the ultraviolet SHG experiments. The fundamental source ranges from $410 \mathrm{~nm}$ to $2200 \mathrm{~nm}$. As shown in Fig. 3c, e, the broadband phase-matching condition was achieved in both samples A and B owing to the aperiodic broken regions and fluctuated reciprocal vectors (Supplementary Figs. S4, S5). However, the strongest output signal is centered at $242 \mathrm{~nm}$ and $214 \mathrm{~nm}$ in samples $A$ and $\mathrm{B}$, respectively, which is consistent with our theory. In sample A, the output power increases with the square of the fundamental wave power, in which the maximum SHG output power $P_{2 \omega}^{A}$ is $38.5 \mu \mathrm{W}$ for an incident power $P_{0}$ of $3.1 \mathrm{~mW}$ (Fig. $3 \mathrm{~d}$ ), relating to a high conversion 

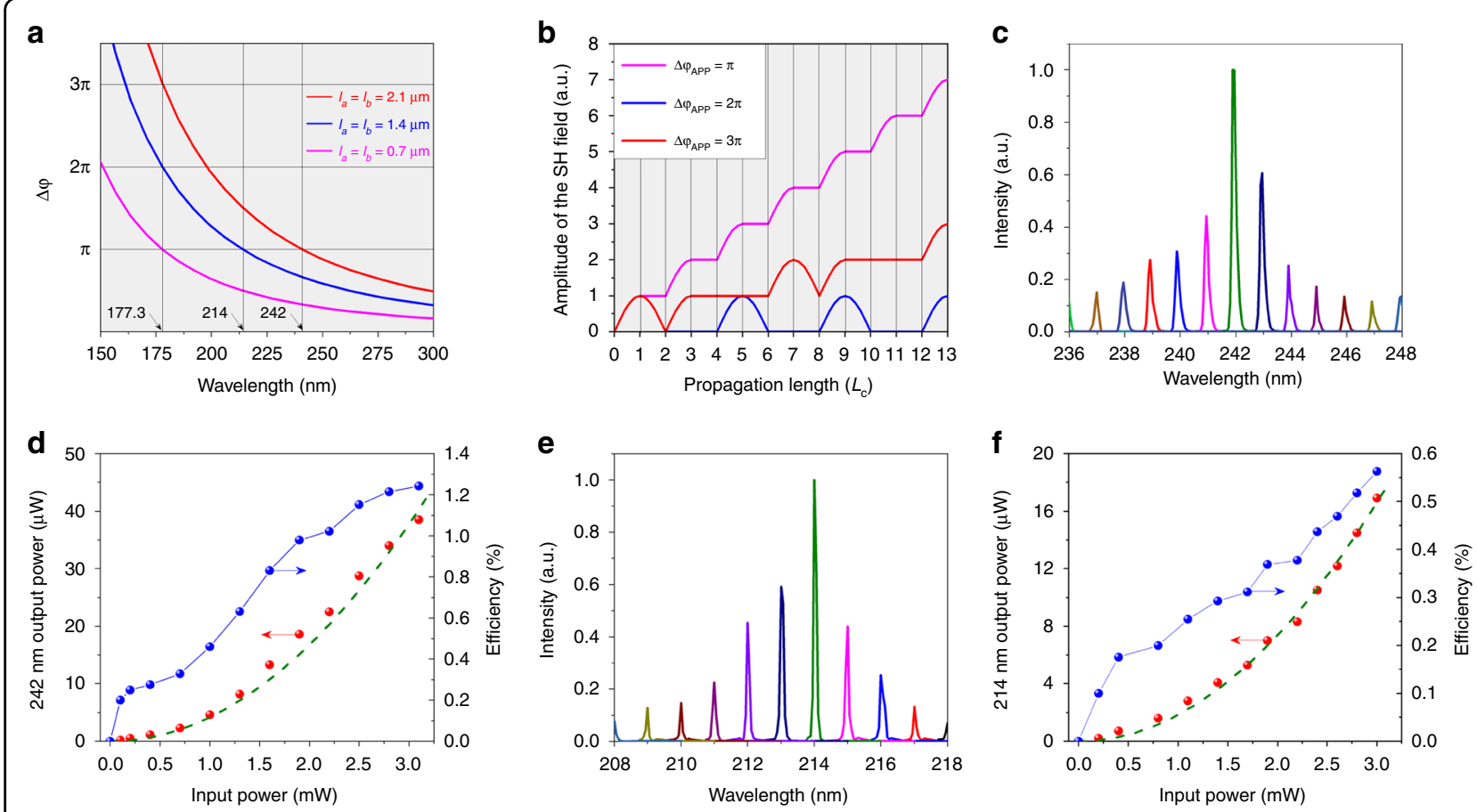

Fig. 3 Experimental demonstration of the APP SHG process in the ultraviolet region. a Theoretical calculation of the APP $\left(\triangle \varphi_{\text {APP }}\right)$ dispersion of the APP quartz samples with $L_{a}=L_{b}=2.1 \mu \mathrm{m}, 1.4 \mu \mathrm{m}$, and $0.7 \mu \mathrm{m}$. b Schematic estimation of the SH field amplitude of the APP quartz with different shifted phases $\left(\triangle \varphi_{\text {APP }}\right)$ for the same crystal length with coherent length $L_{c} . \mathbf{c} S H G$ response at various fundamental wavelengths $(236-248 \mathrm{~nm})$ in APP quartz with $L_{a}=L_{b}=2.1 \mu \mathrm{m}$. d 242-nm SHG output power in quartz (red point) and the optical conversion efficiency (blue point) with $L_{a}=L_{b}=$ $2.1 \mathrm{~mm}$. The green dotted line is the quadratic fitting curve for the SHG output power with an R factor equal to 0.971. e SHG response at various fundamental wavelengths $\left(208-218 \mathrm{~nm}\right.$ ) in APP quartz with $L_{a}=L_{b}=1.4 \mu \mathrm{m}$. $\mathbf{f} 214-\mathrm{nm}$ SHG output power in quartz (red point) and the optical conversion efficiency (blue point) with $L_{a}=L_{b}=1.4 \mu \mathrm{m}$. The green dotted line is the quadratic fitting curve for the SHG output power with an $R$ factor equal to 0.984

efficiency $\eta$ of $1.24 \%$. In addition, in sample B, the output power also depends on the square of the input power of $428 \mathrm{~nm}$. The highest $P_{2 \omega}^{B}$ is $16.9 \mu \mathrm{W}$ at a wavelength of $214 \mathrm{~nm}$ for an incident power $P_{0}$ of $3.1 \mathrm{~mW}$ (Fig. 3f), corresponding to $\eta$ of $0.55 \%$. The slightly reduced efficiency in sample B can be attributed to a lower transmission and larger refractive indices with blueshifted wavelengths. These highly efficient results illuminate that our proposed APP strategy is also valid in the ultraviolet region.

Finally, we attempted to extend the APP strategy into the deep-ultraviolet (DUV) region, an indispensable spectral range for high-resolution photolithography and angular resolution photoelectron spectrometry (ARPES ${ }^{23}$. In particular, 177.3-nm coherent sources have greatly put forward electron state studies in strong correlation systems, such as topological insulators ${ }^{24}$, high-temperature superconductors $^{12}$ and topological semimetals ${ }^{25}$. However, it is indeed difficult to achieve birefringent phase matching in the DUV region owing to the large requisite birefringence (at least 0.07). To date, direct phase-matchable SHG output has only been realized in KBBF crystal ${ }^{16}$. However, the strong layer growth habit and toxic $\mathrm{BeO}$ hinder its further applications in DUV coherent lasers. Despite many reported novel nonlinear optical materials with short absorption cutoffs in the deep-ultraviolet region, these materials could not generate deep-ultraviolet coherent light due to their insufficient birefringence ${ }^{17}$. Therefore, as a "Holy Grail" in the optical community, a $177.3-\mathrm{nm}$ coherent laser still needs to be produced via other technical routes, just like APP.

First, we compared the basic properties of nine DUV transparent nonlinear optical crystals that have a short ultraviolet transparent cutoff wavelength ${ }^{18,26-30}$ to meet the primary requirement for APP crystals, as depicted in Fig. 4a. Among the crystals, only KBBF has demonstrated efficient deep-ultraviolet second-harmonic generation with birefringent phase matching ${ }^{15,16}$. By the comprehensive comparison shown in Supplementary Table S1, it can be found that the quartz and $\mathrm{BPO}_{4}$ should be optimized crystals for demonstrating APP 177.3-nm output in the DUV region. The calculated coherent lengths of these two crystals for the $355-\mathrm{nm}$ SHG process are 0.701 and $0.785 \mu \mathrm{m}^{30}$, respectively. However, it is still difficult to achieve a large $\mathrm{BPO}_{4}$ crystal with a high-optical quality due to its ultrahigh melting viscosity ${ }^{31}$. Therefore, 


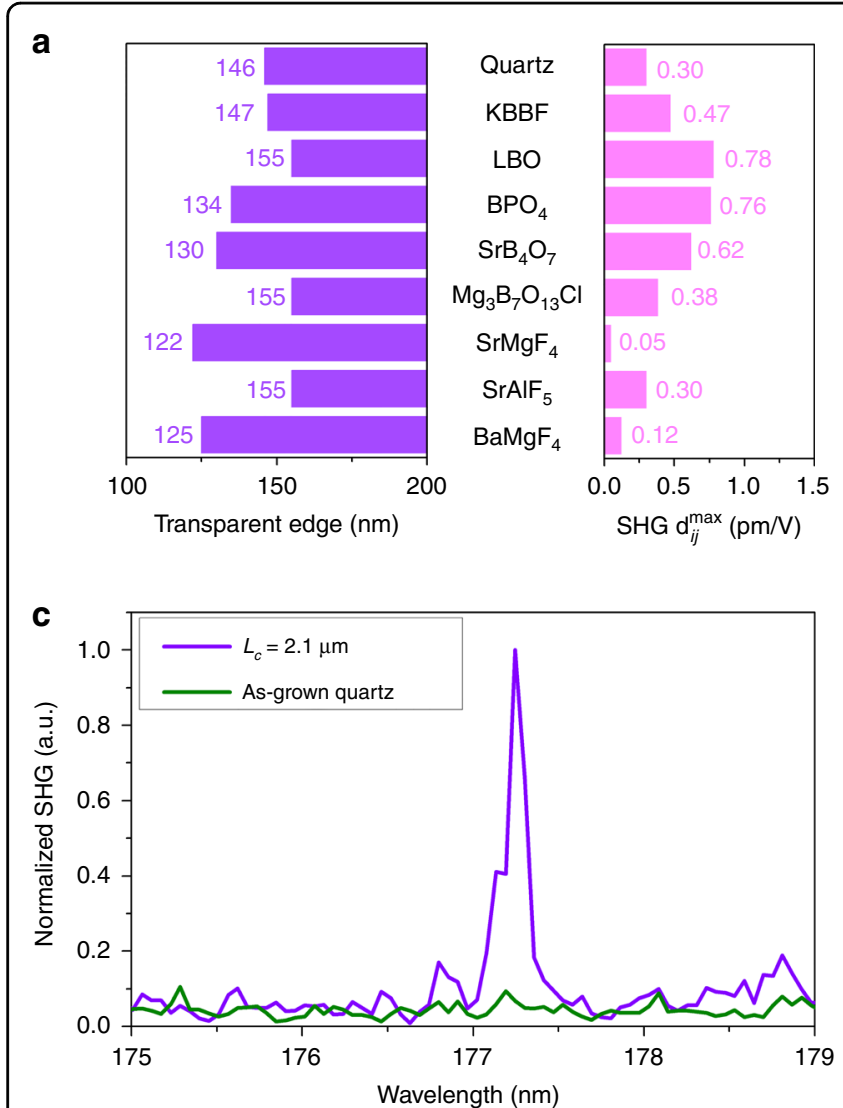

\section{b}

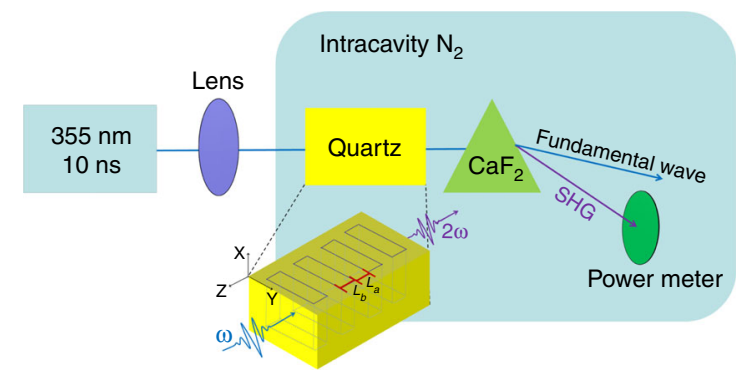

Fig. 4 Experimental demonstration of the APP SHG process in the deep-ultraviolet region. a Comparison of common deep-ultraviolet transparent nonlinear optical crystals (only crystals with a transparent cutoff edge below $160 \mathrm{~nm}$ are plotted). b Experimental setup of the deepultraviolet SHG process from $355 \mathrm{~nm}$ to $177.3 \mathrm{~nm}$. The focal length of the lens is $100 \mathrm{~mm}$. The quartz sample and power meter were placed in a closed chamber filled with pure $\mathrm{N}_{2}$ to avoid SHG 177.3-nm absorption induced by oxygen. A CaF 2 prism was employed to separate the fundamental and SHG signals. c SHG response signal $\left(177.3 \mathrm{~nm}\right.$ ) in APP quartz with $L_{a}=L_{b}=2.1 \mu \mathrm{m}$ (purple solid line) and as-grown quartz (green solid line). d 177.3-nm SHG output power in APP quartz (purple point) with $L_{a}=L_{b}=2.1 \mu m$ and $\Delta \varphi=3 \pi$ and as-grown quartz (green point). The 193-nm output in quasi-phase-matched twined quartz (red point) is adapted from ref. ${ }^{33}$

commercial quartz was selected as the SHG crystal in the present DUV APP experiments. Quartz crystal has a maximum nonlinear coefficient of $d_{11}=0.3 \mathrm{pm} / \mathrm{V}$, comparable to the nonlinear coefficient of KBBF $\left(d_{11}=\right.$ $0.47 \mathrm{pm} / \mathrm{V})^{15}$. The birefringence $\left(n_{\mathrm{e}}-n_{\mathrm{o}}\right)$ of quartz is only 0.01 at $355 \mathrm{~nm}$, leading to impossible birefringent phasematching ${ }^{32}$. In addition, quartz belongs to the nonpolar trigonal 32 system $\left(P 3_{1} 21\right.$ space group, No. 152), indicating that it has no reversal ferroelectric domains to achieve traditional quasi-phase matching. Second, the experimental setup of DUV SHG is also different from that of the early stated visible SHG process (Fig. 4b). Generally, 177.3-nm light will be strongly absorbed by oxygen in air. Therefore, the APP quartz and power meter were placed in a chamber full of nitrogen to eliminate the absorption of oxygen. An additional $\mathrm{CaF}_{2}$ prism was added to deflect and separate the fundamental $(355 \mathrm{~nm})$ and SHG $(177.3 \mathrm{~nm})$ signals. Third, as stated in Fig. 3a, the required period length $L_{\mathrm{a}}$ (and $L_{\mathrm{b}}$ ) for
$\Delta \phi_{\mathrm{APP}}=\Delta \phi_{\mathrm{PD}}=\pi$ of quartz is $0.7 \mu \mathrm{m}$. However, this value is smaller than the minimum accuracy of femtosecond laser manufacturing, as shown in Supplementary Fig. S4. Therefore, APP quartz with $L_{\mathrm{a}}=L_{\mathrm{b}}=2.1 \mu \mathrm{m}$ was utilized to generate a 177.3-nm SHG laser, corresponding to a phase difference of $\Delta \phi_{\mathrm{APP}}=\Delta \phi_{\mathrm{PD}}=3 \pi$. Evidently, this conversion efficiency is lower than that the case for a phase difference of $\pi$. The quartz samples written with an energy intensity of $240 \mathrm{~J} / \mathrm{cm}^{2}$ were used to maintain a high DUV transmittance.

As depicted in Fig. 4c, an anticipated peak located at $177.3 \mathrm{~nm}$ emerges in APP quartz, which represents the first efficient DUV SHG output in quartz. In comparison, there is no detectable SHG signal in the as-grown crystalline quartz, suggesting that our phase-compensating strategy is indeed significant. With an improvement of the incident power $P_{0}$ up to $19 \mathrm{~mW}$, the output SHG power $P_{2 \omega}$ increases to $20.4 \mu \mathrm{W}$, corresponding to an optical conversion efficiency $\eta$ of $1.07 \%$ and a normalized 
conversion efficiency of $\eta_{N}=1.25 \times 10^{-5 \%} / \mathrm{W} / \mathrm{cm}^{2}$. As shown in Fig. 4d, the optical conversion efficiency of the present APP quartz is much higher than that of stressed twin quartz $\left(\eta=0.38 \%, \eta_{N}=2.18 \times 10^{-3 \%} / \mathrm{W} / \mathrm{cm}^{2}\right)$ at the longer wavelength of $193 \mathrm{~nm}$ at the expense of the normalized conversion efficiency ${ }^{33}$. Theoretically, the SHG power should depend on the fundamental power quadratically. The near-linear relationship could be attributed to the additional influence of the beam size, wavelength, absorption, etc. Compared with the results in the visible and ultraviolet ranges, the relatively low conversion efficiency at $177.3 \mathrm{~nm}$ results from the inevitable absorption of the samples and residual oxygen, the loss on the ordered/disordered interface and the large APP of $\Delta \phi_{\text {APP }}$ $=3 \pi$. The output power and conversion efficiency could be further enhanced by reducing the interface loss and realizing $\Delta \phi_{\mathrm{APP}}=\Delta \phi_{\mathrm{PD}}=\pi$ in APP quartz with precise laser direct writing technology. Accordingly, the APP method should be a significant route to obtain deepultraviolet coherent lasers.

In summary, for the first time, a universal additional periodic phase for the phase-matching condition in nonlinear optics has been theoretically proposed, beyond traditional phase matching with natural birefringence of crystals and quasi-phase matching with reversible ferroelectric domains. APP technology is suitable for any acentric crystal (nonpolar, polar, and ferroelectric phases), and among such crystals, a nonpolar nonlinear crystal is the best candidate to demonstrate this theory. Taking $\mathrm{LiNbO}_{3}$ and quartz crystals as examples, an efficient 532$\mathrm{nm}$ laser in the visible region and a 242/214-nm laser in the ultraviolet region were demonstrated by APP SHG conversion. In particular, deep-ultraviolet 177.3-nm generation was first achieved via a periodic disordered quartz crystal (nonpolar phase) with a high efficiency of $1.07 \%$. This APP strategy provides a versatile route for arbitrary nonlinear crystals at broadband wavelengths. Alongside the present SHG in the DUV region, it can be proposed that the APP strategy should also be available for a nonlinear optical process in the infrared region by using SHG, optical parametric oscillation, frequency difference, etc., where the phase difference $\Delta \phi_{\mathrm{PD}}$ could be compensated by the additional phase difference $\Delta \phi_{\text {APP. }}$. More importantly, this order/disorder alignment adds a variable physical parameter into the optical system, thus leading to a next-generation revolution in nonlinear or linear modulation and classical or quantum photonics.

\section{Acknowledgements}

This work was financially supported by the National Natural Science Foundation of China $(51890863,51772173$, and 51632004), the National Key Research and Development Program of China (Grant Nos. 2016YFB0701002 and 2016YFB1 102301), and the Provincial Key Research and Development Program of Shandong (Grant No. 2017CXGC0414).

\section{Author contributions}

H.Y. and H.Z. proposed the concept and designed the experiments. M.S. and F. L. carried out the sample synthesis, characterization and laser experiments. All of the authors contributed to the overall scientific interpretation and edited the manuscript.

\section{Conflict of interest}

The authors declare that they have no conflict of interest.

Supplementary information is available for this paper at https://doi.org/ 10.1038/s41377-020-0281-4.

Received: 25 December 2019 Revised: 28 February 2020 Accepted: 3 March 2020

Published online: 18 March 2020

\section{References}

1. Franken, P. A. et al. Generation of optical harmonics. Phys. Rev. Lett. 7, 118-119 (1961).

2. Boyd, R. W. Nonlinear Optics. 3rd edn. (Academic Press, San Diego, 2008).

3. Petit, Y. et al. Angular quasi-phase-matching. Phys. Rev. A 76, 063817 (2007).

4. Zhu, S. N., Zhu, Y. Y. \& Ming, N. B. Quasi-phase-matched third-harmonic generation in a quasi-periodic optical superlattice. Science 278, 843-846 (1997).

5. Armstrong, J. A. et al. Interactions between light waves in a nonlinear dielectric. Phys. Rev. 127, 1918-1939 (1962).

6. Wei, D. Z. et al. Efficient nonlinear beam shaping in three-dimensional lithium niobate nonlinear photonic crystals. Nat. Commun. 10, 4193 (2019).

7. Chen, X. et al. Quasi-phase matching via femtosecond laser-induced domain inversion in lithium niobate waveguides. Opt. Lett. 41, 2410-2413 (2016).

8. Thomas, J. et al. Quasi phase matching in femtosecond pulse volume structured X-cut lithium niobate. Laser Photonics Rev. 7, L17-L20 (2013).

9. Pan, J. W. et al. Experimental realization of freely propagating teleported qubits. Nature 421, 721-725 (2003).

10. Suchowski, H. et al. Phase mismatch-free nonlinear propagation in optical zero-index materials. Science 342, 1223-1226 (2013).

11. Yan, D. X. et al. High-average-power, high-repetition-rate tunable terahertz difference frequency generation with GaSe crystal pumped by $2 \mu \mathrm{m}$ dualwavelength intracavity KTP optical parametric oscillator. Photonics Res. 5, 82-87 (2017).

12. Meng, J. Q. et al. Coexistence of fermi arcs and fermi pockets in a high- $T_{c}$ copper oxide superconductor. Nature 462, 335-338 (2009).

13. Savage, N. Ultraviolet lasers. Nat. Photonics 1, 83-85 (2007).

14. Jin, S. Q. et al. Note: deep ultraviolet Raman spectrograph with the laser excitation line down to $177.3 \mathrm{~nm}$ and its application. Rev. Sci. Instrum. $\mathbf{8 5}$ 046105 (2014).

15. Chen, C. T. et al. Deep-UV nonlinear optical crystal $\mathrm{KBe}_{2} \mathrm{BO}_{3} \mathrm{~F}_{2}$-discovery growth, optical properties and applications. Appl. Phys. B 97, 9-25 (2009).

16. Togashi, T. et al. Generation of vacuum-ultraviolet Light by an optically contacted, prism-coupled $\mathrm{KBe}_{2} \mathrm{BO}_{3} \mathrm{~F}_{2}$ crystal. Opt. Lett. 28, 254-256 (2003).

17. Halasyamani, P. S. \& Rondinelli, J. M. The must-have and nice-to-have experimental and computational requirements for functional frequency doubling deep-UV crystals. Nat. Commun. 9, 2972 (2018).

18. Trabs, P. et al. Generation of coherent radiation in the vacuum ultraviolet using randomly quasi-phase-matched strontium tetraborate. Opt. Lett. 41, 618-621 (2016).

19. Davis, K. M. et al. Writing waveguides in glass with a femtosecond laser. Opt. Lett. 21, 1729-1731 (1996).

20. Wei, D. Z. et al. Experimental demonstration of a three-dimensional lithium niobate nonlinear photonic crystal. Nat. Photonics 12, 596-600 (2018).

21. Zhang, X. Y. et al. Symmetry-breaking-induced nonlinear optics at a microcavity surface. Nat. Photonics 13, 21-24 (2019).

22. Ishizuki, H. \& Taira, T. Quasi phase-matched quartz for intense-laser pumped wavelength conversion. Opt. Express 25, 2369-2376 (2017).

23. Eismann, U. et al. Short, shorter, shortest: diode lasers in the deep ultraviolet. Laser Focus World 52, 39-44 (2016).

24. Xie, Z. J. et al. Orbital-selective spin texture and its manipulation in a topological insulator. Nat. Commun. 5, 3382 (2014) 
25. LV, B. Q. et al. Observation of three-component fermions in the topological semimetal molybdenum phosphide. Nature 546, 627-631 (2017).

26. Wang, Z. J. et al. $\mathrm{Mg}_{3} \mathrm{~B}_{7} \mathrm{O}_{13} \mathrm{Cl}$ : a new quasi-phase matching crystal in the deepultraviolet region. Adv. Funct. Mater. 28, 1804089 (2018).

27. Villora, E. G. et al. Birefringent- and quasi phase-matching with $\mathrm{BaMgF}_{4}$ for vacuum-UV/UV and mid-IR all solid-state lasers. Opt. Express 17, 12362-12378 (2009).

28. Shimamura, K. et al. Advantageous growth characteristics and properties of $\mathrm{SrAlF}_{5}$ compared with $\mathrm{BaMgF}_{4}$ for UVNUV nonlinear optical applications. J. Cryst. Growth 275, 128-134 (2005).
29. Yelisseyev, A. P. et al. Structures and optical properties of two phases of $\mathrm{SrMgF}_{4}$. Phys. Chem. Chem. Phys. 17, 500-508 (2015).

30. Zhang, $X$. et al. Determination of the coherence length in the vacuumultraviolet spectral region for the $\mathrm{BPO}_{4}$ crystal. J. Opt. 14, 035204 (2012).

31. Zhao, S. G. et al. Growth, thermophysical and electrical properties of the nonlinear optical crystal $\mathrm{BPO}_{4}$. Cryst. Res. Technol. 47, 391-396 (2012).

32. Ghosh, G. Dispersion-equation coefficients for the refractive index and birefringence of calcite and quartz crystals. Opt. Commun. 163, 95-102 (1999).

33. Kurimura, S. et al. Quartz revisits nonlinear optics: twinned crystal for quasiphase matching [Invited]. Optical Mater. Express 1, 1367-1375 (2011). 